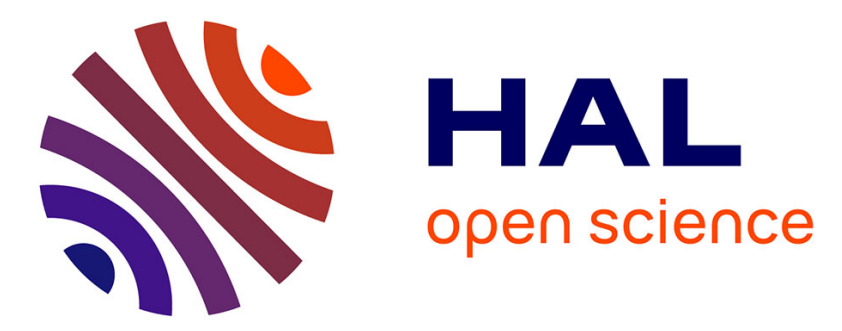

\title{
State Observation of LTV Systems with Delayed Measurements: A Parameter Estimation-based Approach with Fixed Convergence Time
}

\author{
Alexey Bobtsov, Nikolay Nikolaev, Romeo Ortega, Denis Efimov
}

\section{To cite this version:}

Alexey Bobtsov, Nikolay Nikolaev, Romeo Ortega, Denis Efimov. State Observation of LTV Systems with Delayed Measurements: A Parameter Estimation-based Approach with Fixed Convergence Time. Automatica, 2021, 10.1016/j.automatica.2021.109674 . hal-03174995v2

\section{HAL Id: hal-03174995 \\ https://inria.hal.science/hal-03174995v2}

Submitted on 21 Mar 2021

HAL is a multi-disciplinary open access archive for the deposit and dissemination of scientific research documents, whether they are published or not. The documents may come from teaching and research institutions in France or abroad, or from public or private research centers.
L'archive ouverte pluridisciplinaire HAL, est destinée au dépôt et à la diffusion de documents scientifiques de niveau recherche, publiés ou non, émanant des établissements d'enseignement et de recherche français ou étrangers, des laboratoires publics ou privés. 


\title{
State Observation of LTV Systems with Delayed Measurements: A Parameter Estimation-based Approach with Fixed Convergence Time
}

\author{
Alexey Bobtsov ${ }^{\text {d,a }}$, Nikolay Nikolaev ${ }^{\mathrm{a}}$, Romeo Ortega ${ }^{\mathrm{b}, \mathrm{a}}$, Denis Efimov ${ }^{\mathrm{c}}$. \\ ${ }^{a}$ Department of Control Systems and Robotics, ITMO University, Kronverkskiy av. 49, Saint-Petersburg, 197101, Russia \\ ${ }^{\mathrm{b}}$ Departamento Académico de Sistemas Digitales, ITAM, Ciudad de México, México \\ ' INRIA, Univ. Lille, CNRS, UMR 9189 - CRIStAL, F-59000 Lille, France \\ ${ }^{\mathrm{d}}$ Hangzhou Dianzi University (HDU), China
}

\begin{abstract}
In this paper we address the problem of state observation of linear time-varying (LTV) systems with delayed measurements, which has attracted the attention of many researchers - see [9] and references therein. We show that, the parameter estimationbased observer (PEBO) design proposed in $[4,5]$ provides a very simple solution to the problem with reduced prior knowledge. Moreover, when PEBO is combined with the dynamic regressor extension and mixing (DREM) estimation technique [1,6], the estimated state converges in fixed-time with extremely weak excitation assumptions.
\end{abstract}

Key words: Linear time-varying systems; state observer; delay systems; parameter estimation.

\section{Introduction and Problem Formulation}

It is common in control applications that the measuring devices introduce time-varying delays. This fact complicates the problem of estimating the state of a dynamical system since the output signal will be subject to delays, a problem that has been studied by many authors. For linear time invariant (LTI) systems, such a difficulty is well understood and the convergence of the observer is usually verified checking the feasibility of a linear matrix inequality [2]. On the other hand, the problem for LTV systems is widely open - see the literature review and the list of references in the recent papers $[7,9]$.

We consider in this paper an LTV system

$$
\begin{aligned}
& \dot{x}(t)=A(t) x(t)+B(t) u(t) \\
& y(t)=C(\varphi(t)) x(\varphi(t))
\end{aligned}
$$

\footnotetext{
* This paper was not presented at any IFAC meeting. Corresponding author N. Nikolaev. Tel. +79213090016.

Email addresses: bobtsov@mail.ru (Alexey Bobtsov), nanikolaev@itmo.ru (Nikolay Nikolaev), romeo.ortega@itam.mx (Romeo Ortega), denis.efimov@inria.fr (Denis Efimov).
}

where $x(t) \in \mathbb{R}^{n}, u(t) \in \mathbb{R}^{m}, y(t) \in \mathbb{R}^{q}, \varphi(t)$ is a continuous known nonnegative delay function that codifies the measurement delay, that is

$$
\varphi(t):=t-d(t)
$$

where $d(t)$ is the time-varying delay verifying

$$
0 \leq d(t) \leq d_{M}
$$

and the state initial condition $x(0)=x_{0} \in \mathbb{R}^{n}$.

All system matrices are continuous and known with $C(\varphi(t))$ bounded and $A(t), B(t)$ verifying the following.

Assumption 1 The state transition matrix of the homogeneous part of the system $(1)$, denoted $\Phi(t, \tau)$, verifies

$$
\|\Phi(t, \tau)\| \leq c_{1}, \forall t \geq \tau .
$$

\section{Assumption 2}

$$
\int_{\tau}^{t}\|\Phi(t, \tau) B(\tau)\| d \tau \leq c_{2}, \forall t \geq \tau
$$


Remark 1 Assumption 1 is equivalent to uniform stability of the homogeneous part of the system (1) [8, Theorem 6.4], while Assumption 2 is a necessary and sufficient condition for bounded-input-bounded-state stability of (1) [8, Theorem 12.2].

In this paper we design an observer

$$
\begin{aligned}
& \dot{\chi}(t)=F(\chi(t), u(t), y(t)) \\
& \hat{x}(t)=H(\chi(t), u(t), y(t))
\end{aligned}
$$

with $\chi(t) \in \mathbb{R}^{n_{\chi}}$ such that all signals are bounded and fixed convergence time (FCT) of the estimated state is ensured, that is,

$$
\hat{x}(t)=x(t), \forall t \geq t_{c},
$$

for some $t_{c} \in(0, \infty)$ and for all $x_{0} \in \mathbb{R}^{n}, \chi(0) \in \mathbb{R}^{n_{\chi}}$ and all continuous bounded $u(t)$.

\section{Main result}

To streamline the presentation of our observer we recall from [3] the following.

Definition 1 A bounded signal $\Delta(t) \in \mathbb{R}$ is an interval exciting (IE) if there exists a time $t_{\mathrm{IE}} \in(0, \infty)$ such that

$$
\int_{0}^{t_{\mathrm{IE}}} \Delta^{2}(s) d s \geq \rho
$$

for some $\rho>0$.

Proposition 1 Consider the system (1) satisfying Assumptions 1 and 2 and $d(t)$ verifying (2) with the generalized $\mathrm{PEBO}+\mathrm{DREM}$

$$
\begin{aligned}
\dot{\xi}(t) & =A(t) \xi(t)+B(t) u(t) \\
\dot{\Phi}_{A}(t) & =A(t) \Phi_{A}(t), \Phi_{A}(0)=I_{n} \\
\dot{Y}(t) & =-\lambda Y(t)+\lambda \Psi^{\top}(t) z(t) \\
\dot{\Omega}(t) & =-\lambda \Omega(t)+\lambda \Psi^{\top}(t) \Psi(t)
\end{aligned}
$$

with $\lambda>0$ and the gradient parameter estimator ${ }^{1}$

$$
\dot{\hat{\theta}}(t)=-\gamma \Delta(t)[\Delta(t) \hat{\theta}(t)-\mathcal{Y}(t)]
$$

with $\gamma>0$, and the definitions

$$
\begin{aligned}
\Psi(t) & :=C(\varphi(t)) \Phi_{A}(\varphi(t)) \\
z(t) & :=C(\varphi(t)) \xi(\varphi(t))-y(t) \\
\mathcal{Y}(t) & :=\operatorname{adj}\{\Omega(t)\} Y(t) \\
\Delta(t) & :=\operatorname{det}\{\Omega(t)\},
\end{aligned}
$$

\footnotetext{
1 See [10, Chapter 2] for the definition of a gradient estimator.
}

where $\operatorname{adj}\{\cdot\}$ is the adjugate matrix. Define the state estimate as

$$
\hat{x}(t)=\xi(t)-\Phi_{A}(t) \frac{1}{1-w_{c}(t)}\left[\hat{\theta}(t)-w_{c}(t) \hat{\theta}(0)\right],
$$

with

$$
\dot{w}(t)=-\gamma \Delta^{2}(t) w(t), w(0)=1,
$$

and $w_{c}(t)$ defined via the clipping function

$$
w_{c}(t)=\left\{\begin{array}{ll}
w(t) & \text { if } \quad w(t) \leq 1-\mu \\
1-\mu & \text { if } w(t)>1-\mu
\end{array},\right.
$$

where $\mu \in(0,1)$ is a designer chosen parameter. Assume $\Delta(t)$ verifies (4) with

$$
\rho=-\frac{1}{\gamma} \ln (1-\mu)
$$

Then, (3) is ensured for some $t_{c} \geq t_{\mathrm{IE}}$, and all signals remain bounded.

PROOF. First, we prove the signal boundedness claim. Due to Assumptions 1 and 2 and boundedness of $u(t)$, $\xi(t)$ and the fundamental matrix $\Phi_{A}(t)$ are bounded. This, together with boundedness of $C(\varphi(t))$, guarantees that $z(t)$ and $\Psi(t)$ are bounded. Finally, positivity of $\lambda$ ensures that $Y(t)$ and $\Omega(t)$ are also bounded.

Second, we apply the PEBO technique to derive a (vector) linear regression equation (LRE). For this purpose, define the error signal $e(t):=\xi(t)-x(t)$, which satisfies

$$
\dot{e}(t)=A(t) e(t)
$$

hence

$$
e(t)=\Phi_{A}(t) \theta
$$

with $\theta:=e(0)$. Consequently,

$$
x(t)=\xi(t)-\Phi_{A}(t) \theta .
$$

The output of the system (1) then satisfies

$$
y(t)=C(\varphi(t))\left[\xi(\varphi(t))-\Phi_{A}(\varphi(t)) \theta\right] .
$$

From which we get a LRE, that allows us to identify $\theta$, as

$$
z(t)=\Psi(t) \theta
$$

where we used the definitions in (7a) and (7b). Notice that these signals are well defined because the assumption (2) ensures that $\varphi(t)$ is lower bounded.

Third, we apply the DREM procedure to (12) to generate $n$ scalar LREs. Towards this end, we consider the 
following chain of implications

$$
\begin{aligned}
(12) & \Rightarrow \Psi^{\top}(t) z(t)=\Psi^{\top}(t) \Psi(t) \theta \quad\left(\Leftarrow \Psi^{\top}(t) \times\right) \\
& \Rightarrow Y(t)=\Omega(t) \theta\left(\Leftarrow \frac{\lambda}{\mathbf{p}+\lambda}[\cdot] \text { and }(5 \mathrm{c}),(5 \mathrm{~d})\right) \\
& \Rightarrow \mathcal{Y}(t)=\Delta(t) \theta, \quad(\Leftarrow \operatorname{adj}\{\Omega\} \times \text { and }(7 \mathrm{c}),(7 \mathrm{~d})),
\end{aligned}
$$

with $\mathbf{p}=\frac{d}{d t}$, where we have used the fact that for any, possibly singular, $n \times n$ matrix $L$ we have $\operatorname{adj}\{L\} L=$ $\operatorname{det}\{L\} I_{n}$ in the last line.

The last step is the analysis of the gradient estimator (6) with the FCT observer (8)-(10). Replacing the latter identity in (6) yields the error equation

$$
\dot{\tilde{\theta}}(t)=-\gamma \Delta^{2}(t) \tilde{\theta}(t)
$$

where $\tilde{\theta}(t):=\hat{\theta}(t)-\theta$. The solution of this equation is given by

$$
\tilde{\theta}(t)=e^{-\gamma \int_{0}^{t} \Delta^{2}(s) d s} \tilde{\theta}(0) .
$$

Notice that the solution of $(9)$ is

$$
w(t)=e^{-\gamma \int_{0}^{t} \Delta^{2}(s) d s},
$$

hence we have that

$$
\tilde{\theta}(t)=w(t) \tilde{\theta}(0)
$$

Clearly, this is equivalent to

$$
[1-w(t)] \theta=\hat{\theta}(t)-w(t) \hat{\theta}(0) .
$$

On the other hand, if $\Delta(t)$ is IE with $\rho$ satisfying (11), we have that there exists a $t_{c} \geq t_{0}$ such that

$$
w(t)=w_{c}(t)<1, \forall t \geq t_{c} .
$$

Consequently, we conclude that

$$
\frac{1}{1-w_{c}(t)}\left[\hat{\theta}(t)-w_{c}(t) \hat{\theta}(0)\right]=\theta, \forall t \geq t_{c} .
$$

Replacing this identity in (8) completes the proof.

Remark 2 Note that the upper bound on the delay implicitly impacts the value of $t_{c}$ given in (4), thus, it predefines the fixed time of convergence.

Remark 3 Another, more complex, solution to this problem that requires strict positivity of the delay $d(t)$ and the knowledge of its derivative is reported in [9] under the classical assumption of existence of an exponentially stable Luenberger observer for the LTV system (1) with $\varphi(t)=t$, i.e. [9, Assumption 2]. The estimator is based on - the now classical-PDE representation of the delay, with an observer designed for the coupled LTV-PDE system.
Remark 4 In [7] a Kalman-Bucy-like observer with fractional powers is proposed, which ensures that the state estimation enters a residual set in a finite time provided the pair $(C(t), A(t))$ is uniformly completely observable. As it is well known [8], this assumption is a sufficient condition for the verification of $[9$, Assumption 2]. It is obvious that both assumptions are strictly stronger than our IE condition.

\section{Simulation Results}

Consider the LTV system (1) with $m=q=1, n=2$ and

$$
A=\left[\begin{array}{cc}
0 & 1 \\
-\sin ^{2}(t) & 0
\end{array}\right], B=\left[\begin{array}{l}
0 \\
1
\end{array}\right], C=\left[\begin{array}{l}
1 \\
0
\end{array}\right]
$$

For simulations consider the initial conditions $x(0)=$ $\operatorname{col}(1,2)$ and zero for all the other states.

We consider three cases:

C1 $\varphi(t)=t$ (Fig. 1 and Fig. 2);

C2 $\varphi(t)=\varphi(t-\tau), \tau=1+0.9 \sin (t)$ (Fig. 3 and Fig. 4$)$;

C3 $\varphi(t)=\varphi(t-\tau), \tau=0.1+\cos ^{2}(3 t)$ (Fig. 5 and Fig. $6)$.

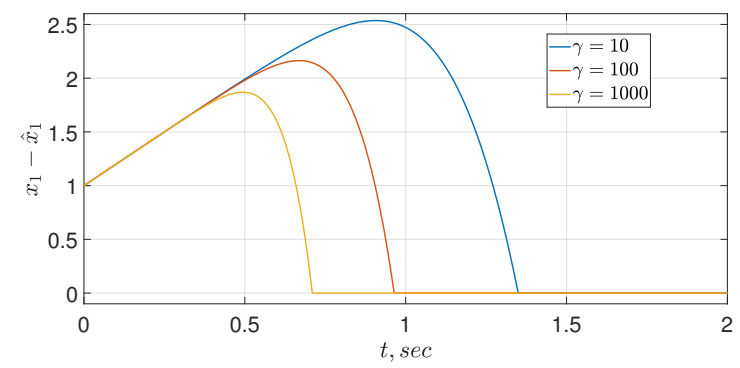

Fig. 1. Error transients $x_{1}(t)-\hat{x}_{1}(t)$ for different $\gamma$ and case C1

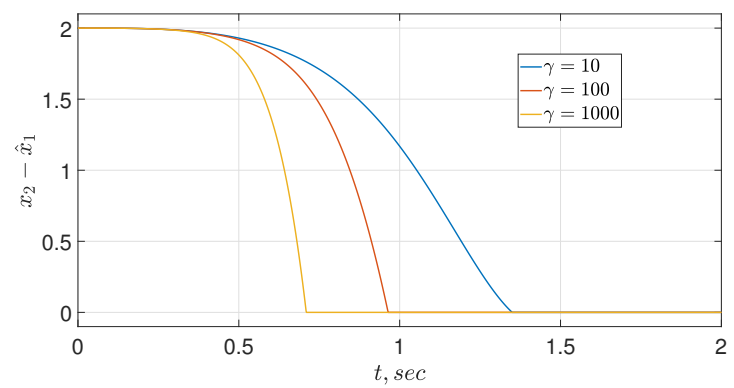

Fig. 2. Error transients $x_{2}(t)-\hat{x}_{2}(t)$ for different $\gamma$ and case C1

\section{Conclusions and Future Research}

A state observer for LTV systems with delayed measurements of the form (1) has been proposed. The main feature of the observer is that, thanks to the use of DREM, 


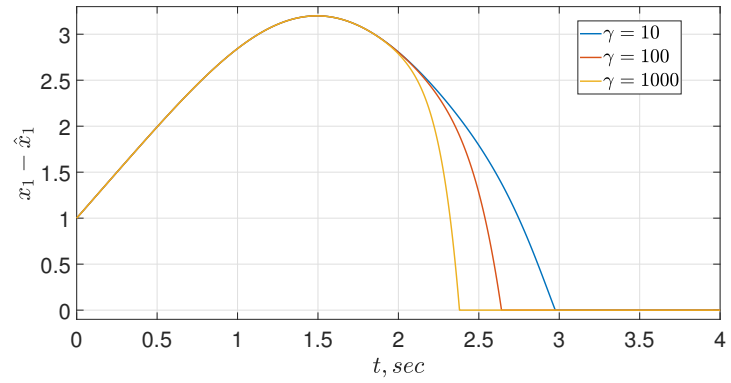

Fig. 3. Error transients $x_{1}(t)-\hat{x}_{1}(t)$ for different $\gamma$ and case C2

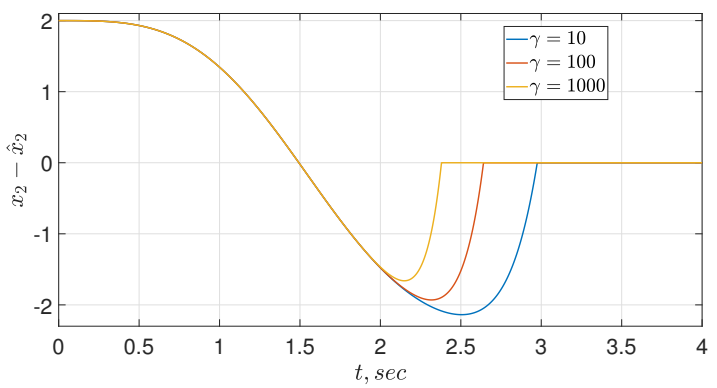

Fig. 4. Error transients $x_{2}(t)-\hat{x}_{2}(t)$ for diffrerent $\gamma$ and case C2

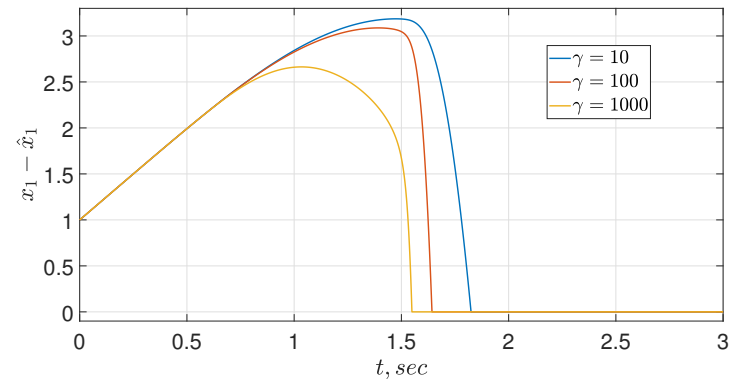

Fig. 5. Error transients $x_{1}(t)-\hat{x}_{1}(t)$ for diffrerent $\gamma$ and case C3

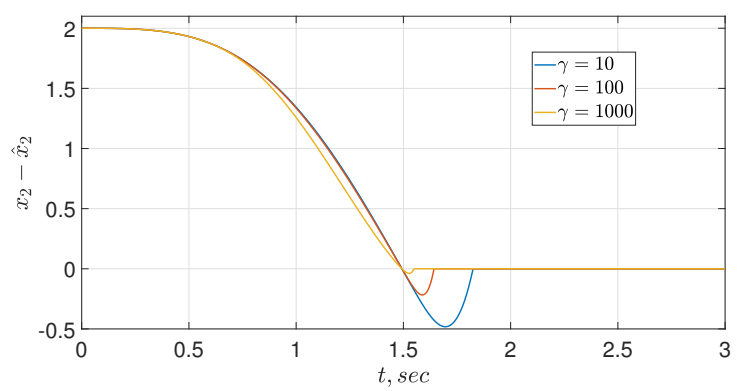

Fig. 6. Error transients $x_{2}(t)-\hat{x}_{2}(t)$ for diffrerent $\gamma$ and case C3

it ensures convergence in fixed time under the extremely weak assumption of IE. Following the PEBO approach, the observation of the state is carried out via the estima- tion of some suitable initial conditions and, in contrast to [9], we do not require any kind of "delay compensation". To the best of our knowledge this is the strongest result available for this problem.

Current research is under way to relax the strict assumption of exact knowledge of the system dynamics and the time delay function.

Acknowledgements: The authors would like to express their gratitude to the anonymous reviewers that provided insightful remarks that were instrumental to improve the quality of the paper. This work was supported by the Ministry of Science and Higher Education of Russian Federation, passport of goszadanie no. 20190898 .

\section{References}

[1] Stanislav Aranovskiy, Alexey Bobtsov, Romeo Ortega, and Anton Pyrkin. Performance enhancement of parameter estimators via dynamic regressor extension and mixing. IEEE Transactions on Automatic Control, 62(7):3546-3550, 2016. (See also arXiv:1509.02763 for an extended version.).

[2] Emilia Fridman. Introduction to time-delay systems: Analysis and control. Birkhäuser Basel, 2014.

[3] Gerhard Kreisselmeier and Gudrun Rietze-Augst. Richness and excitation on an interval-with application to continuoustime adaptive control. IEEE Transactions on Automatic Control, 35(2):165-171, 1990.

[4] Romeo Ortega, Alexey Bobtsov, Nikolay Nikolaev, Johannes Schiffer, and Denis Dochain. Generalized parameter estimation-based observers: Application to power systems and chemical-biological reactors. arXiv preprint arXiv:2003.10952, 2020.

[5] Romeo Ortega, Alexey Bobtsov, Anton Pyrkin, and Stanislav Aranovskiy. A parameter estimation approach to state observation of nonlinear systems. Systems \& Control Letters, 85:84-94, 2015.

[6] Romeo Ortega, Dmitry N Gerasimov, Nikita E Barabanov, and Vladimir $\mathrm{O}$ Nikiforov. Adaptive control of linear multivariable systems using dynamic regressor extension and mixing estimators: Removing the high-frequency gain assumptions. Automatica, 110:108589, 2019.

[7] Juan G Rueda-Escobedo, Rosane Ushirobira, Denis Efimov, and Jaime A Moreno. Gramian-based uniform convergent observer for stable ltv systems with delayed measurements. International Journal of Control, 93(2):226-237, 2020.

[8] Wilson J Rugh. Linear System Theory. Prentice-Hall, Inc., 1996.

[9] Ricardo Sanz, Pedro Garcia, and Miroslav Krstic. Observation and stabilization of LTV systems with timevarying measurement delay. Automatica, 103:573-579, 2019.

[10] Shankar Sastry and Marc Bodson. Adaptive control: Stability, Convergence and Robustness. Prentice Hall, USA, 1989. 